\title{
Fuzzy-Aided Syntactic Scene Analysis ${ }^{\star}$
}

\author{
Marzena Bielecka ${ }^{1}$ and Marek Skomorowski ${ }^{2}$ \\ ${ }^{1}$ Department of Goeinformatics and Applied Computer Science, \\ AGH University of Science and Technology, \\ Al. Mickiewicza 30, 30-059 Kraków, Poland \\ bielecka@agh.edu.pl \\ ${ }^{2}$ Institute of Computer Science, Jagiellonian University, \\ Nawojki 11, 30-072 Kraków, Poland \\ skomorowski@ii.uj.edu.pl
}

\begin{abstract}
In syntactic pattern recognition a pattern can be described by a graph. The problem of recognition is to determine if a pattern, represented by a describing graph, belongs to a language $L(G)$, generated by a graph grammar $G$. The so-called $I E$ graphs are used for pattern description. They are generated by so-called ETPL $(k)$ graph grammars. The purpose of this paper is to present an idea of a new approach to syntactic recognition of fuzzy patterns represented by fuzzy IE graphs, followed the example of random IE graphs. This methodology can be used in embodied multi-agent systems for a scene analysis.
\end{abstract}

Keywords: Syntactic pattern recognition, distorted patterns, graph grammars.

\section{Introduction}

Agents are entities capable of taking account of what surrounds them. In referring to an embodied cognitive multi-agent system this means, among others, that agents are managed to analyze the scene they act on. In particular, an agent has got a symbolic and explicit representation of the surrounding world ([2], page 17). Syntactic pattern recognition based on graphs is one of the classical approach to this task.

In a node replacement graph grammar, a node of a derived graph is replaced by a new subgraph, which is connected to the remainder of the graph. A node replacement is controlled by a production of a given graph grammar. An example of a node replacement graph grammar is an ETPL $(k)$ (embedding transformationpreserving production-ordered $k$-left nodes unambiguous) grammars introduced in [3]. The so-called IE (indexed edge-unambiguous) graphs have been defined in [3] for a description of patterns (scenes) in syntactic pattern recognition. Nodes in an $I E$ graph denote pattern primitives. Edges between two nodes in an $I E$ graph represent spatial relations between pattern primitives. An idea of a probabilistic improvement of syntactic recognition of distorted patterns represented

\footnotetext{
^ This work was partially supported by the AGH grant number 1010140461.
} 
by graphs is described in [4] and [6]. A random IE graph approach $([4,6])$ is proposed for such a description and an efficient parsing algorithm for $I E$ graphs (the computational complexity is $O\left(n^{2}\right)$ ) is presented in [6].

In this paper we present an idea of approach to syntactic recognition by fuzzy $I E$ graphs, followed the example of random $I E$ graphs. Fuzziness allows us describe in proper way patterns that can not be presented univocally.

\section{Fuzzy IE Graphs for Fuzzy Patterns Representation}

Let us remind definition of an $I E$ graph ([3]). An indexed edge-unambiguous graph, an $I E$ graph over $\Sigma$ and $\Gamma$ is a quintuple

$$
g=(V, E, \Sigma, \Gamma, \Phi)
$$

where

$V$ - is a finite, nonempty set of nodes to which indices have been ascribed in an unambiguous way,

$\Sigma$ - is a finite, nonempty set of node labels,

$\Gamma$ - is a finite, nonempty set of edge labels,

$E$ - is a set of edges of the form $(v, \lambda, w), v, w \in V, \lambda \in \Gamma$, such that index of $v$ is less than index of $w$

$\Phi: V \rightarrow \Sigma$ - is a node labeling function.

Assume that both labeled objects in nodes of a graph and spatial relations are represented by fuzzy sets of a first order with membership functions $\mu_{i}$ and $\nu_{i}$ respectively. Let, furthermore, the set of all objects $\Sigma$ be $n$-elemental and the set of all spatial relations be $k$-elemental. Let us define, informally, a fuzzy IE graph as an $I E$ graph in which nodes labels are replaced by a vector $\mu=\left[\mu_{1}, \ldots, \mu_{n}\right]$ of values of membership functions $\mu_{i}, i \in\{1, \ldots, n\}$ and edges labels are replaced by vector $\nu=\left[\nu_{1}, \ldots, \nu_{k}\right]$ of values of membership functions $\nu_{j}, j \in\{1, \ldots, k\}$ see Fig.1.

The fuzzy measure of an outcome $I E$ graph, obtained form a given fuzzy IE graph, is equal to the value of a $T$-norm $T$ of the values components of the node and edge vectors. An axiomatic definition of $T$-norms is given in [5], definition 4.22 , page 80 . Having a fuzzy $I E$ graph $R$ the fuzzy measure $\lambda_{r}$ of an outcome graph $r$ is calculated as

$$
\lambda_{r}=T\left(\stackrel{P}{T}\left(\mu_{f_{r}(p)}^{p}\right), \stackrel{S}{T}\left(\nu_{g_{r}(s)}^{s}\right)\right)
$$

where

$p$ - is a number of a regarded node,

$s$ - is a number of an edge,

$f_{r}(p)$ - is a chosen component number of a vector $\mu^{p}$ whereas

$g_{r}(s)$ - is a chosen number of component of a vector $\nu^{s}$. 
If the arithmetic product is used as a $T$-norm then the presented parsing algorithm (see Section 3) is identical to the random parsing algorithm described in $[6]$.

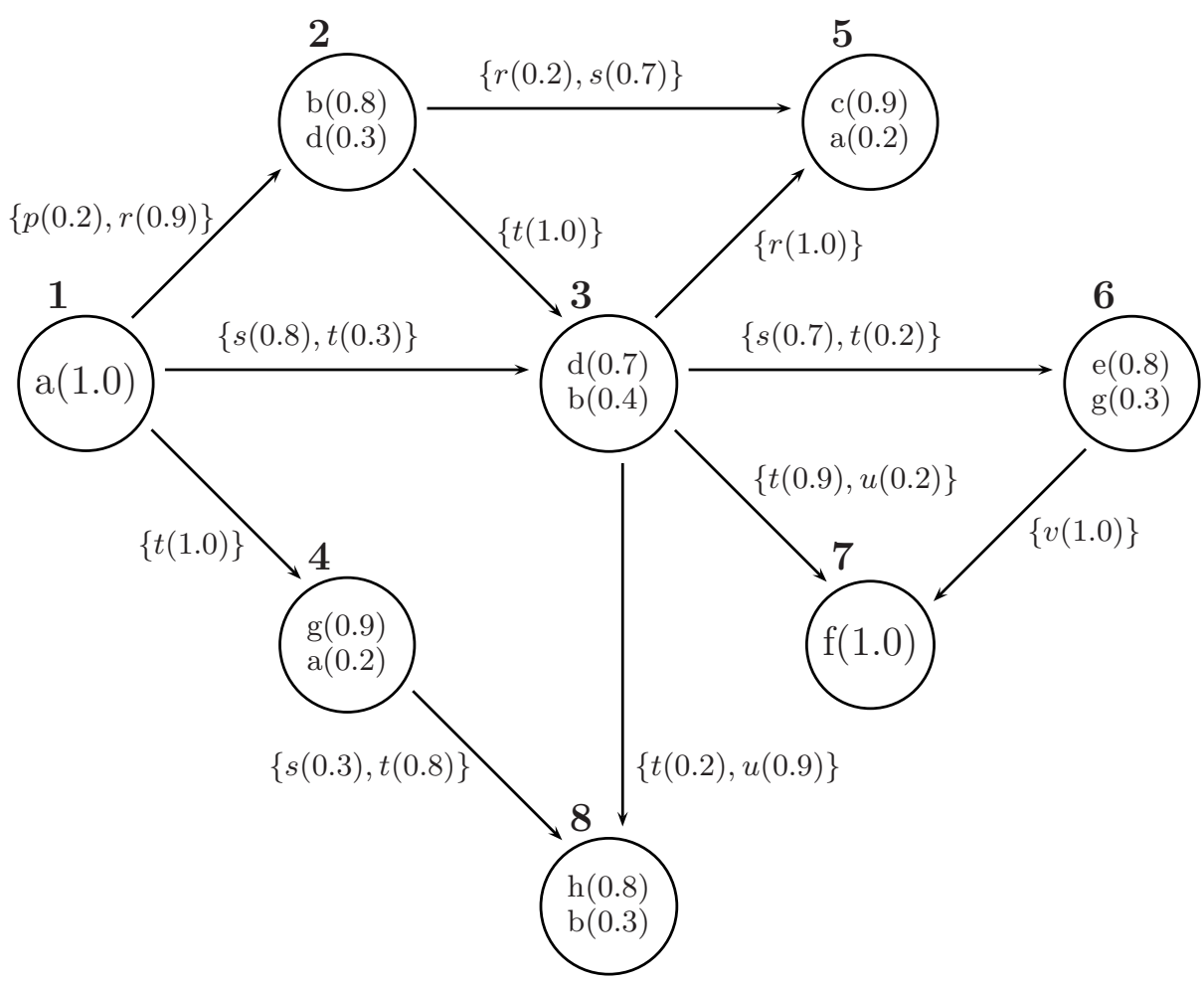

Fig.1. An example of fuzzy $I E$ graph representing an unknown pattern

\section{Parallel Parsing of Fuzzy IE Graphs}

Given an unknown pattern represented by a fuzzy $I E$ graph $R$, the problem of recognition of a pattern under study is to determine if an outcome $I E$ graph $r$, obtained from the fuzzy $I E$ graph $R$, belongs to a graph language $L(G)$ generated by an $E T P L(k)$ graph grammar $G$. In the proposed parallel and cut-off strategy of fuzzy IE graph parsing a number of simultaneously derived graphs is equal to a certain number limit. In this case, derived graphs spread through the search tree, but only the best, that is with maximum measure value, limit graphs are expanded.

Let us introduce the following notations: $Z$ - a starting graph of an $\operatorname{ETPL}(k)$ graph grammar $G, P$ - is a set of productions of the grammar $G, R$ - an analyzed fuzzy graph. The idea of the proposed parsing algorithm is the following: 
1. Apply to the starting graph $Z$ productions belonging to the set $P$, which are admissible for further derivations. If such productions do not exist then stop the parsing algorithm.

2. For subgraphs obtained in the point 1, compute values of their membership functions using a given $T$-norm.

3. For further derivation choose a number (limit) of derived subgraphs with the biggest values of membership function.

4. To the chosen subgraphs apply these productions from $P$ which are admissible for further derivations. If such productions do not exist then stop the parsing algorithm.

5. Repeat the points 3 and 4 until fuzzy-outcome graph $R$ is obtained.

6. Stop the parsing algorithm.

An example of the introduced algorithm application can be found in [1].

\section{Concluding Remarks}

In this paper we have proposed an idea of a new approach to recognition of fuzzy patterns represented by graphs. To take into account variations of a fuzzy pattern under study, a description of the analyzed pattern based on fuzzy sets of the first order was introduced. The fuzzy IE graph has been proposed here for such a description. It should be stressed that informal introduction of this class of graphs has been forced by the volume limitation of the paper. The parsing algorithm, having the computational complexity $O\left(n^{2}\right)$, presented in $[3,6]$, is extended in such a way that fuzzy patterns, represented by fuzzy IE graphs, can be recognized. In the algorithm a $T$-norm is used for calculation of value of membership measure of output graphs. Such solution makes that the algorithm is very flexible. In particular, if arithmetic product is used as a $T$-norm then the algorithm is the same as the random one described in [6].

\section{References}

1. Bielecka M., Skomorowski M., Bielecki A.: Fuzzy-syntactic approach to pattern recognition and scene analysis, under revision.

2. Ferber J.: Multi-Agent Systems. An Introducing to Distributed Artificial Intelligence. Addison-Wesley, Harlow (1999)

3. Flasiński M.: On the parsing of deterministic graph languages for syntactic pattern recognition. Pattern Recognition, Vol. 26 (1993) 1-16

4. Flasiński M., Skomorowski, M.: Parsing of random graph languages for automated inspection in statistical-based quality assurance systems. Machine Graphics and Vision, Vol. 7 (1998) 565 - 623

5. Rutkowski L.: Artificial Intelligence Techniques and Methods. PWN, Warszawa (2005) (in Polish)

6. Skomorowski, M.: Use of random graph parsing for scene labeling by probabilistic relaxation. Pattern Recognition Letters, Vol. 20 (1999) 949-956 